\title{
Diabetes mellitus, glycemic traits, and cerebrovascular disease: a Mendelian randomization study
}

Short title: Genetically determined glycemic traits and stroke

\author{
Marios K. Georgakis, $\mathrm{MD}^{1,2} *$, Eric L Harshfield, $\mathrm{PhD}^{3} *$, Rainer Malik, $\mathrm{PhD}^{1}$, Nora \\ Franceschini, MD, MPH ${ }^{4}$, Claudia Langenberg, $\mathrm{MD}, \mathrm{PhD}^{5}$, Nicholas J. Wareham, MD, $\mathrm{PhD}^{5}$, \\ Hugh S. Markus, DM, F Med $\mathrm{Sci}^{3} \dagger$, Martin Dichgans, $\mathrm{MD}^{1,6,7} \dagger$
}

\footnotetext{
${ }^{1}$ Institute for Stroke and Dementia Research, University Hospital, Ludwig-Maximilians-University, Munich, Germany

${ }^{2}$ Graduate School for Systemic Neurosciences, Ludwig-Maximilians-University, Munich, Germany

${ }^{3}$ Stroke Research Group, Department of Clinical Neurosciences, University of Cambridge, Cambridge, UK

${ }^{4}$ Department of Epidemiology, UNC Gillings Global School of Public Health, Chapel Hill, NC, USA

${ }^{5}$ MRC Epidemiology Unit, University of Cambridge, Cambridge, UK

${ }^{6}$ Munich Cluster for Systems Neurology (SyNergy), Munich, Germany.

${ }^{7}$ German Centre for Neurodegenerative Diseases (DZNE), Munich, Germany.

* these authors contributed equally to the manuscript

$\uparrow$ these authors contributed equally to the manuscript
}

\section{Corresponding Author:}

Martin Dichgans, MD

Institute for Stroke and Dementia Research, University Hospital

Ludwig-Maximilians-University (LMU)

Feodor-Lynen-Str. 17, 81377 Munich, Germany

Phone: +49-89-4400-46018; Fax: +49-89-4400-46040

E-mail: martin.dichgans@med.uni-muenchen.de

Total word count: 7,276 (title page, abstract, text, references, tables, figure legends)

Subject terms: Diabetes Type 2; Cerebrovascular Disease/Stroke; Ischemic Stroke; Intracranial Hemorrhage; Genetic, Association Studies 


\section{ABSTRACT}

Rationale: Type 2 diabetes mellitus (T2D) is an established risk factor for cerebrovascular disease but the mechanisms underlying this association remain elusive. Disentangling the causal effects of T2D, hyperglycemia, and pre-diabetic phenotypes (insulin resistance, $\beta$-cell dysfunction) on major etiological stroke subtypes (ischemic stroke, intracerebral hemorrhage, ischemic stroke subtypes) could inform the development of preventive strategies.

Objective: We employed Mendelian randomization (MR) to explore the effects of genetic predisposition to T2D, hyperglycemia, insulin resistance, and $\beta$-cell dysfunction on risk of stroke subtypes and related cerebrovascular phenotypes.

Methods and Results: We selected instruments for genetic predisposition to T2D, HbA1c levels, fasting glucose levels, insulin resistance, and $\beta$-cell dysfunction (proxied by pro-insulin levels) based on published genome-wide association studies (up to 898,130 individuals). Applying two-sample MR, we examined associations with ischemic stroke, intracerebral hemorrhage, and ischemic stroke subtypes (large artery, cardioembolic, small vessel stroke; up to 60,341 cases and 454,450 controls). We further explored associations with the related phenotypes of carotid atherosclerosis, imaging markers of cerebral white matter integrity, and brain atrophy. Genetic predisposition to T2D and elevated $\mathrm{HbA1c}$ levels in the pre-diabetic range were associated with higher risk of any ischemic stroke, large artery stroke, carotid plaque and small vessel stroke. Independently of HbA1c levels, we further found genetic predisposition to insulin resistance to be associated with large artery and small vessel stroke, whereas predisposition to $\beta$-cell dysfunction was associated with small vessel stroke. Predisposition to $\beta$-cell dysfunction was further associated with intracerebral hemorrhage, lower grey matter volume, and total brain volume.

Conclusions: This study supports causal effects of T2D and hyperglycemia on large artery and small vessel stroke. We show differential effects of genetically determined insulin resistance and $\beta$-cell dysfunction on large artery and small vessel stroke that might have implications for anti-diabetic treatments targeting these mechanisms. 


\section{INTRODUCTION}

Cerebrovascular disease is a major public health issue ${ }^{1}$ ranking as the second leading cause of mortality and adult disability worldwide. ${ }^{2,3}$ Type 2 diabetes mellitus (T2D) is an established risk factor for cerebrovascular disease. ${ }^{4,5}$ In cohort studies, T2D shows associations with higher risk for both ischemic and hemorrhagic stroke independently of other risk factors. ${ }^{6}$ Also, several studies found associations of measures of hyperglycemia (glycated hemoglobin (HbA1c) and fasting glucose levels) with risk of stroke, both in patients with and without diabetes. ${ }^{6,7}$ However, large-scale randomized controlled trials (RCTs) testing intensive glucose-lowering in patients with T2D show no significant reductions in risk of stroke, possibly due to insufficient power. ${ }^{8-11}$ Moreover, the effects of T2D or hyperglycemia on etiological stroke subtypes (large artery stroke, cardioembolic stroke, small vessel stroke, intracerebral hemorrhage) remain elusive.

Currently available anti-diabetic medications act by either directly lowering glucose levels or by targeting two major mechanisms that contribute to hyperglycemia: insulin resistance or pancreatic $\beta$ cell dysfunction. ${ }^{12,13}$ Observational data suggest that markers of insulin resistance, $\beta$-cell dysfunction, and hyperglycemia influence the risk of cardiovascular disease independently of each other. ${ }^{14,15}$ However, data on stroke and its etiological subtypes are lacking. Moreover, there is a risk of confounding in observational studies. Developing targeted strategies for stroke prevention in patients at risk or suffering from T2D would require disentangling these relationships.

Mendelian randomization (MR) may help to clarify these associations. MR uses genetic variants as instruments for traits of interest and is not prone to confounding and reverse causation. ${ }^{16}$ As such, MR has proven to be a powerful methodology for inferring causality. ${ }^{17,18}$ The availability of largescale genome-wide association studies (GWAS) with detailed phenotyping of cases further enables the exploration of etiological stroke subtypes that are typically not considered in epidemiological studies.

Here, we leveraged data from large-scale GWASs and performed MR analyses, with the following aims: (i) to examine the effects of genetic predisposition to T2D on risk of ischemic stroke, ischemic stroke subtypes, and intracerebral hemorrhage; (ii) to explore the effects of genetically determined 
measures of hyperglycemia (HbA1c and fasting glucose levels) among diabetes-free individuals on these phenotypes; (iii) to disentangle the associations of genetic predisposition to insulin resistance, $\beta$-cell dysfunction, and hyperglycemia with major stroke etiologies; and (iv) to explore associations between diabetic traits and related vascular phenotypes including carotid atherosclerosis, neuroimaging markers of white mater integrity, and brain atrophy.

\section{METHODS}

\section{Study design and data sources}

This is a two-sample MR study following the guidelines for strengthening the reporting of Mendelian randomization studies (STROBE-MR). ${ }^{19}$ The data sources that we used are detailed in Table 1. The study is based on publicly available summary statistics from GWAS consortia. Our study design and the phenotypes explored in these analyses are depicted in Figure 1. We explored associations of genetic predisposition to T2D, measures of hyperglycemia (HbA1c and fasting glucose levels), as well as markers of insulin resistance and $\beta$-cell dysfunction with cerebrovascular disease phenotypes including stroke subtypes, carotid atherosclerosis, white matter (WM) integrity, and brain atrophy. Information on all genetic variants used as instruments in the current study are presented in Supplementary Tables S1-S6.

\section{Genetic instrument selection}

Diabetes mellitus type 2. We selected genetic instruments from the latest GWAS meta-analysis for T2D based on 74,124 cases and 824,006 controls of European ancestry from 32 studies included in the DIAGRAM consortium. ${ }^{20}$ The analyses were adjusted for age, sex, and population structure. We selected as genetic instruments all the 403 distinct genetic variants showing significant associations with $\mathrm{T} 2 \mathrm{D}$ in this meta-analysis (either genome-wide significance at $\mathrm{p}<5 \times 10^{-8}$ or independent locuswide significance at $\mathrm{p}<10^{-5}$ at significant loci; Supplementary Table S1). In sensitivity analyses, we restricted our genetic instruments to those reaching genome-wide significance. 
Hyperglycemia. We selected genetic instruments for HbA1c levels (per 1\%-increment) based on a GWAS that we performed on 408,989 diabetes-free individuals of White British ancestry in the UK Biobank (UKB). ${ }^{21}$ We excluded individuals with self-reported history of physician-diagnosed diabetes, use of oral antidiabetic drugs or insulin, $\mathrm{HbA} 1 \mathrm{c}$ level $>6.5 \%$, or random glucose levels $>200 \mathrm{mg} / \mathrm{dl}$. We adjusted for age, sex, genotyping platform array, assessment center, and the first 20 principal components of the population structure and performed the analyses using BOLT-LMM with correction for relatedness and subtle population stratification. For fasting glucose levels (per 1SD increment), we used the most recent GWAS meta-analysis (adjusted only for age, sex, and population structure) by the MAGIC consortium on 133,010 diabetes-free individuals of European ancestry. ${ }^{22}$ For both HbA1c and fasting glucose, we selected as instruments genetic variants reaching genome-wide significance $\left(\mathrm{p}<5 \times 10^{-8}\right)$ after pruning for linkage disequilibrium at an $\mathrm{r}^{2}<0.01$ threshold. We identified 543 instruments for HbA1c levels and 21 for fasting glucose levels (Supplementary Tables S2-S3). ${ }^{22}$

As several genetic variants may influence HbA1c levels through effects on erythrocyte biology and not by inducing hyperglycemia, ${ }^{23}$ to isolate the effects of the hyperglycemia-related genetic component of HbAlc levels, we performed sensitivity analyses excluding those variants reported to be associated at $\mathrm{p}<0.001$ with erythrocyte-related traits in Phenoscanner ${ }^{24}$ (hemoglobin concentration, red blood cell count, hematocrit, mean corpuscular volume, mean corpuscular hemoglobin concentration, mean corpuscular hemoglobin, red cell distribution width, reticulocyte count, reticulocyte fraction of red cells, immature fraction of reticulocytes, high light scatter percentage of red cells, high light scatter reticulocyte count).

Insulin resistance and p-cell dysfunction. As instruments for insulin resistance we used 53 genetic variants identified in a multi-trait GWAS to associate with all three components of this phenotype (fasting insulin levels, triglycerides and HDL-cholesterol; Supplementary Table S4). ${ }^{25} \mathrm{We}$ weighted the instruments based on their effects on fasting insulin levels (per 1-log increment) in a GWAS meta-analysis of 108,557 diabetes-free European individuals. ${ }^{22}$ In accordance with existing literature, we proxied $\beta$-cell dysfunction based on fasting proinsulin levels (per 1 log-increment). ${ }^{26-28}$ 
We used summary statistics from a GWAS meta-analysis of 16,378 diabetes-free European individuals and identified 21 genetic instruments (at $\mathrm{p}<5 \times 10^{-8}$ and $\mathrm{r}^{2}<0.01$; Supplementary Table S5). ${ }^{26}$ The GWAS for fasting insulin levels was adjusted for age, sex, and population structure, ${ }^{22}$ whereas the GWAS for pro-insulin was additionally adjusted for fasting insulin levels. ${ }^{26}$

We further used T2D-associated genetic variants previously grouped into clusters of diabetic endophenotypes; three clusters of insulin resistance (related to obesity, fat distribution, or lipid metabolism) and two clusters of $\beta$-cell dysfunction both associated with reduced levels of fasting insulin, but with opposing effects on fasting proinsulin. ${ }^{29}$ We used the clusters of the variants and the respective weights per variant and cluster, as described in the study by Udler et al. (Supplementary

Table S6). ${ }^{30}$

\section{Proportion of explained variance and instrument strength}

For all genetic variants used as instruments, we estimated the proportion of explained variance for the respective phenotypes (Supplementary Tables S1-S5). We estimated the variance explained by each genetic variant for T2D based on the method by So et al. for binary phenotypes ${ }^{31}$ and for the continuous traits we used a previously described formula based on summary statistics. ${ }^{32}$ For the estimations regarding T2D, we used a prevalence rate of $8.5 \%$, according to the 2015 estimate of the global prevalence of the disease by the International Diabetes Federation. ${ }^{33}$ We then calculated the Fstatistic as an indicator of instrument strength. ${ }^{34}$ In sensitivity analyses, we restricted our selection to variants with $\mathrm{F}>10$, which is widely considered to indicate instruments with very low probability for weak instrument bias. ${ }^{34}$

\section{Associations with outcomes}

We then examined associations of the selected instruments with ischemic stroke, ischemic stroke subtypes, and intracerebral hemorrhage (ICH) as the primary outcomes of interest. For ischemic stroke, we used summary GWAS data from the MEGASTROKE trans-ethnic population, mainly 
consisting of European individuals $(70 \%) .{ }^{35,36}$ We extracted summary GWAS statistics for any ischemic stroke (60,341 cases, 451,210 controls) and for the major ischemic stroke subtypes according to the TOAST (Trial of Org 10172 in Acute Stroke Treatment) classification: ${ }^{37}$ large artery stroke (6,688 cases, 238,513 controls), cardioembolic stroke (9,006 cases, 352,852 controls), and small vessel stroke (11,710 cases, 287,067 controls). GWAS data for ICH were derived from the International Stroke Genetics Consortium (ISGC) GWAS meta-analysis including 1,545 cases and 1,481 controls of European ancestry. ${ }^{38}$

Presence of carotid plaque, markers of WM tract integrity (WM hyperintensities (WMH) volume, mean diffusivity, fractional anisotropy), and markers of brain atrophy (grey matter volume, total brain volume) were explored as secondary outcomes. Carotid plaque data were derived from a GWAS meta-analysis (21,540 cases, 26,894 controls of European ancestry) from the CHARGE consortium. ${ }^{39}$ For the imaging phenotypes (WMH volume, mean diffusivity, fractional anisotropy, grey matter volume, total brain volume), we undertook GWAS analyses in the UK Biobank neuroimaging dataset including 17,534 individuals of White British ancestry based on the MRI sequences, as has been previously described. ${ }^{40}$ We performed linear regression analyses (additive models) for ln-transformed WMH volume, the first principal components of all measurements of mean diffusivity and fractional anisotropy across the different white matter tracts in the diffusion sequences, and for normalized grey matter and total brain volumes. Adjustments were made for age, sex, mean resting and task functional MRI head motion, the genotype platform array, and the first 10 principal components of the population structure.

\section{Statistical analysis}

All analyses were performed in R (v3.5.0; The R Foundation for Statistical Computing) using the MendelianRandomization, TwoSampleMR, and the MR-PRESSO packages.

Main analyses. We applied two-sample MR using association estimates derived from the abovementioned sources. Following extraction of the SNP-specific association estimates between the instruments and the outcomes, and harmonization of the direction of estimates by effect alleles, we 
computed MR estimates for each instrument with the Wald estimator. ${ }^{41}$ We calculated standard errors with the Delta method. We then pooled individual MR estimates using random-effects inverse-variance weighted (IVW) meta-analyses. ${ }^{41}$ For the main analyses, we corrected for multiple comparisons with the false discovery rate (FDR) approach and set statistical significance at qvalue $<0.05$. Associations not reaching this threshold, but showing an unadjusted $\mathrm{p}<0.05$ were considered of nominal significance.

Assessment of pleiotropy and sensitivity analyses. MR estimates derived from the IVW approach could be biased in the presence of directional horizontal pleiotropy. As a measure of overall pleiotropy, we assessed heterogeneity across the SNP-specific MR estimates in the IVW MR analyses with the Cochran's Q statistic (statistical significance set at $\mathrm{p}<0.05$ ). ${ }^{42}$ We further applied alternative MR methods which are more robust to pleiotropic variants. The weighted median estimator allows the use of invalid instruments as long as at least half of the instruments used in the MR analysis are valid. ${ }^{43}$ The MR-Egger regression allows for the estimation of an intercept term that can be used as an indicator of unbalanced directional pleiotropy. ${ }^{44}$ MR-Egger provides less precise estimates and relies on the assumption that the strengths of potential pleiotropic instruments are independent of their direct associations with the outcome. ${ }^{44}$ The intercept obtained from MR-Egger regression was used as a measure of unbalanced pleiotropy ( $<<0.05$ indicated significance).$^{44}$ Finally, MR-PRESSO regresses the SNP-outcome estimates against the SNP-exposure estimates to test for outlier SNPs. ${ }^{45}$ Outliers are detected by sequentially removing all variants from the analyses and comparing the residual sum of squares as a global measure of heterogeneity $(\mathrm{p}<0.05$ for detecting outliers); outliers are then removed and outlier-corrected estimates are provided. ${ }^{45}$ MR-PRESSO still relies on the assumption that at least half of the variants are valid instruments. ${ }^{45}$

Mediation analyses and multivariable Mendelian randomization. We additionally performed MRbased network mediation analyses ${ }^{46}$ to examine the extent to which increases in $\mathrm{HbA1c}$ levels mediated the effects of insulin resistance and $\beta$-cell dysfunction on stroke subtypes. We performed this analysis only for large artery and small vessel stroke, for which we found robust evidence of associations with $\mathrm{HbA1c}$. We calculated the total effects of insulin resistance and $\beta$-cell dysfunction 
on the outcomes of interest using random-effects IVW MR analyses. For the direct effects, we combined the instruments for insulin resistance or $\beta$-cell dysfunction with those for $\mathrm{HbA1c}$ levels and performed multivariable MR analyses for their associations with the stroke subtypes. ${ }^{47}$ To calculate the indirect effects, we calculated the difference between the direct and the total effect estimates. ${ }^{48}$ We finally estimated the proportion of the overall effect of insulin resistance or $\beta$-cell dysfunction on stroke subtypes that was mediated through increases in $\mathrm{HbA1C}$ by dividing the indirect by the total effects. ${ }^{48}$ Standard errors were derived using the delta method.

\section{RESULTS}

The 403 genetic variants used as genetic instruments for T2D explained 17.2\% of the variance in T2D prevalence (Supplementary Table S1), whereas variants used as instruments for the continuous hyperglycemia traits, insulin resistance (proxied by fasting insulin levels), and $\beta$-cell dysfunction (proxied by fasting proinsulin), explained lower proportions of variance: $1.9 \%$ for $\mathrm{HbA1c}, 1.5 \%$ for fasting glucose, $0.7 \%$ for insulin resistance, and $4.5 \%$ for $\beta$-cell dysfunction (Supplementary Tables S1-S5).

\section{Genetic predisposition to type 2 diabetes mellitus and risk of stroke}

In the primary IVW MR analyses, genetic predisposition to T2D (1-log-increment=2.72-fold higher odds) was significantly associated with a higher risk of any ischemic stroke (OR: 1.10, 95\%CI: 1.071.12), large artery stroke (OR: 1.19, 95\%CI: 1.14-1.26), and small vessel stroke (OR: 1.14, 95\%CI: 1.10-1.19; Figure 2). In addition, there was an association of nominal significance with higher risk of cardioembolic stroke (OR: 1.05, 95\%CI: 1.01-1.09). All SNPs used as insturments for genetic predisposition to T2D had F-statitics $>10$, thus indicating a very low probability of weak instrument bias. In sensitivity analyses restricted to SNPs reaching genome-wide significance $\left(\mathrm{p}<5 \times 10^{-8}\right)$ for association with T2D the results remained stable and statistically significant and we further found an association of nominal significance with ICH (Supplementary Table S7). 
With the exception of ICH, there was evidence of significant heterogeneity in all of the main analyses ( $<<0.05$; Supplementary Table S8), but no evidence of unbalanced pleiotropy, as assessed by the Egger intercept p-values (all p>0.05; Supplementary Table S7). Across sensitivity analyses based on alternative MR methods (weighted median, MR-Egger, outlier-corrected MR-PRESSO), all effects remained directionally consistent and all estimates stable with $\mathrm{p}<0.05$ for any ischemic stroke, large artery stroke, and small vessel stroke (Supplementary Table S7).

\section{Genetic predisposition to measures of hyperglycemia and risk of stroke}

In analyses of hyperglycemia traits we found that genetically determined HbAlc levels (per 1\%increment) among diabetes-free individuals were significantly associated with risk of any ischemic stroke (OR: 1.28, 95\%CI: 1.12-1.46), large artery stroke (OR: 1.61, 95\%CI: 1.22-2.13), and small vessel stroke (OR: 1.83, 95\%CI: 1.45-1.33; Figure 3). In contrast, we found no significant associations between genetically determined fasting glucose levels and risk of stroke subtypes (Figure 3).

There was evidence of heterogeneity in the analyses for HbA1c levels (Supplementary Table S8) and in MR-Egger the effect estimates for any ischemic stroke, large artery stroke, and small vessel stroke were smaller (Supplementary Table S7). However, in sensitivity analyses that excluded SNPs influencing HbA1c levels through erythrocyte-related traits, the association estimates were even larger (ischemic stroke, OR: 1.60, 95\%CI: 1.30-1.98; large artery stroke, OR: 2.05, 95\%CI: 1.29-3.26; small vessel stroke, OR: 2.44, 95\%CI: 1.64-3.65) and there was no evidence of heterogeneity (all p>0.10). Similarly, the results remained stable, when excluding SNPs with an Fstatistic $<10$ that might introduce weak instrument bias in the analyses.

\section{Genetic predisposition to insulin resistance, $\beta$-cell dysfunction, and risk of stroke}

We next selected genetic variants as instruments for insulin resistance and $\beta$-cell dysfunction, the two primary underlying mechanisms contributing to the development of hyperglycemia and T2D. 
Among diabetes-free individuals, we found genetic predisposition to insulin resistance (1-log increment in fasting insulin levels) to be associated with a higher risk for ischemic stroke (OR: 1.33, 95\%CI: 1.13-1.57), large artery stroke (OR: 1.60, 95\%CI: 1.12-2.31), and small vessel stroke (OR: 1.63, 95\%CI: 1.21-2.20; Figure 4A). Genetic predisposition to $\beta$-cell dysfunction (1-log increment in fasting proinsulin levels) was further associated with a higher risk for both small vessel stroke (OR: $1.38,95 \% \mathrm{CI}: 1.17-1.63)$ and ICH (OR: $1.75,95 \% \mathrm{CI}: 1.21-2.52)$. There was no heterogeneity in these analyses (Supplementary Table S8) and the results were consistent in alternative MR analyses (Supplementary Table S7).

To gain additional insights in the relationship between insulin resistance, $\beta$-cell dysfunction, and etiological stroke subtypes, we further explored the effects of T2D-associated variants clustered in five different mechanisms of action. These included three clusters for insulin resistance (mediated by obesity, fat distribution, lipid metabolism) and two clusters related to $\beta$-cell dysfunction (associated with high or low proinsulin). In multivariable analyses including all clusters and also adjusting for their effects on $\mathrm{HbA1c}$, we found significant effects of genetic predisposition to $\beta$-cell dysfunction related to high proinsulin on risk of ischemic stroke and small vessel stroke (Figure 4B). We further found genetic predisposition to insulin resistance mediated through altered fat distribution to be associated with higher risk of small vessel stroke. Genetic predisposition to insulin resistance mediated through obesity showed associations of nominal significance with large artery and cardioembolic stroke.

We next explored whether the effects of genetic predisposition to insulin resistance and $\beta$-cell dysfunction on large artery and small vessel stroke are mediated through hyperglycemia (Figure 5). Genetic predisposition to insulin resistance was associated with higher risk of large artery stroke independently of its effects on HbA1c; the indirect effect through increases in HbAlc levels was not significant. For small vessel stroke, we found genetic predisposition to both insulin resistance and $\beta$ cell dysfunction to contribute to a higher risk independently of HbA1c levels; again, the indirect effects through $\mathrm{HbA1c}$ were not significant. HbA1c was associated with a higher risk of large artery and small vessel stroke, independently of insulin resistance or $\beta$-cell dysfunction (Figure 5). 


\section{Genetic predisposition to type 2 diabetes and glycemis traits and associations with etiologically related cerebrovascular phenotypes}

Table 2 presents the MR associations of genetic predisposition to T2D, measures of hyperglycemia, insulin resistance, and $\beta$-cell dysfunction, with carotid plaque, as well as with neuroimaging traits related to white matter integrity and brain atrophy. Genetic predisposition to T2D and genetically elevated $\mathrm{HbA1c}$ levels were associated with carotid plaque. We further found a significant association between genetic predisposition to T2D and lower fractional anisotropy, a diffusion imaging marker of impaired white matter tract integrity, as well as nominally significant associations with lower grey matter and total brain volumes (Table 2). Genetic predisposition to $\beta$-cell dysfunction (1-log increment in fasting proinsulin levels) was further associated with lower grey matter volume (beta: $-0.13,95 \%$ CI: -0.20 to -0.07 ) and total brain volume (beta: $-0.17,95 \% \mathrm{CI}$ : -0.23 to -0.11; Table 2). These results remained stable in sensitivity analyses (Supplementary Table S7).

\section{DISCUSSION}

Levaraging large-scale GWAS data in MR analyses, we investigated the causal associations between $\mathrm{T} 2 \mathrm{D}$, glycemic traits, and cerebrovascular disease. We found genetic predisposition to T2D and hyperglycemia (elevated $\mathrm{HbA1c}$ levels) to be associated with a higher risk of ischemic stroke, particularly large artery and small vessel stroke. Independently of hyperglycemia, genetic predisposition to insulin resistance but not $\beta$-cell dysfunction was associated with higher risk of large artery stroke, whereas genetic predisposition to both insulin resistance and $\beta$-cell dysfunction was associated with small vessel stroke. Genetic determinats for T2D and hyperglycemia further showed significant effects on carotid plaque and fractional anisotropy, a WM neuroimaging marker related to cerebral small vessel disease. Conversely, genetic predisposition to $\beta$-cell dysfunction was associated with intracerebral hemorrhage and neuroimaging markers of brain atrophy.

Our MR results provide genetic evidence for a causal effect of T2D, and also hyperglycemia in the non-diabetic range, on risk of ischemic stroke. While T2D is among the established risk factors for 
stroke and vascular disease in general, ${ }^{5}$ primary prevention trials focusing on intensive glucose control or specific oral anti-diabetic agents showed inconsistent effects on stroke risk. ${ }^{8-11}$ Previous Mendelian randomization studies were underpowered to detect effects of hyperglycemia (HbA1c or fasting glucose levels) on stroke risk. ${ }^{49,50}$ Here, by using data from $>400,000$ individuals from the UK Biobank, we were able to show that genetically elevated HbA1c levels are associated with a higher risk of ischemic stroke, thus suggesting that preventive strategies focusing on long-term HbA1c-lowering will result in risk reductions for ischemic stroke. The lack of significant effects in previous trials might relate to insufficient power due to the low number of incident stroke events, short follow-up periods, and differences in the efficacy profiles of the individual treatments. ${ }^{51}$

We found the effects of genetic predisposition to T2D and hyperglycemia to be specific for large artery and small vessel stroke. In accordance with these results, we found genetic predisposition to T2D to be associated with carotid plaque, an atherosclerotic phenotype, and fractional anisotropy, a marker of WM integrity associated with small vessel disease. Thus, our findings provide evidence for a causal involvement of T2D and hyperglycemia in both large artery atherosclerosis and cerebral small vessel disease. On the contrary, we found no significant effects of T2D or other diabetic traits on cardioembolic stroke. Differences in the magnitude of the effects between stroke subtypes might in part explain the heterogeneity in the effects of glucose-lowering treatments across previous clinical trials. ${ }^{51}$ On the basis of our findings, future trials testing glucose-lowering approaches should account for stroke subtypes.

As another major finding, we show that genetic predisposition to insulin resistance and $\beta$-cell dysfunction influences the risk of stroke independently of hyperglycemia. This could have clinical implications for oral anti-diabetic medications. While all anti-diabetic agents lower glucose levels, some drug classes primarily target insulin sensitivity whereas others primarily target $\beta$-cell function. ${ }^{12,13}$ Our findings indicate that the mechanism of action might have relevance for stroke prevention on top of the glucose-lowering effects. This could further provide another layer of explanation for the heterogeneous effects of existing clinical trials testing different anti-diabetic medications for stroke prevention. ${ }^{52}$ 
Interestingly, we also found differential effects of genetic predispostion to insulin resistance and $\beta$ cell dysfunction on risk of stroke subtypes and related phenotypes. Genetic predisposition to insulin resistance showed effects on both large artery and small vessel stroke indicating more widespread effects on cerebral circulation similar to those of hyperglycemia. In contrast, genetic predisposition to $\beta$-cell dysfunction was associated with small vessel stroke, intracereral hemorrhage, and brain atrophy, which are all manifestations of cerebral small vessel disease. ${ }^{53}$ Given the lack of specific preventive strategies for cerebral small vessel disease, our MR results are of great interest and suggest that strategies targeting $\beta$-cell dysfunction should be explored in future studies.

Our study has several methodological strengths. The large sample size $(898,130$ individuals for diabetic traits and up to 514,791 individuals for stroke) and nature of our datasets provided the power to detect differential effects of diabetes on etiological stroke subtypes and to perform multiple sensitivity analyses for testing the validity of the MR assumptions, thus minimizing the possibility of biased results. While the genetic determinants of $\mathrm{HbAlc}$ might influence its levels via both erythrocyte and glycemic biology, we provided support for the latter, as the effects were stronger when focusing on variants not associated with erythrocyte traits. Incorporating insulin resistance and $\beta$-cell dysfunction on top of hyperglycemia in the analyses offered deeper insights into the pathophysiological mechanisms linking diabetes with the different stroke subtypes. Finally, the exploration of additional cerebrovascular disease traits enabled us to triangulate our findings for stroke subtypes by showing similar associations for etiologically related phenotypes.

Our study also has limitations. First, by design MR examines the effects of lifetime exposure to the traits of interest, which might differ from the effects of clinical interventions (e.g. glucose-lowering approaches) applied for shorter time periods later in life. Second, it was only possible to use proxies for the genetic predisposition to insulin resistance and $\beta$-cell dysfunction. However, available GWASs for biomarkers more closely related to these phenotypes (e.g. the homeostatic model assessment indexes for insulin resistance and $\beta$-cell dysfunction) were underpowered, thus precluding the identification of valid genetic instruments. ${ }^{54}$ Third, the MR analyses for insulin resistance were weighted based on the effects of the genetic variants on fasting insulin adjusting for 
BMI and the analyses for $\beta$-cell dysfunction based on the effects of the variants on fasting proinsulin adjusting for fasting insulin. These adjustments in the original GWASs might increase the risk for collider bias in MR analyses, ${ }^{55}$ which should be considered when interpreting our findings. Fourth, despite using the largest currently available GWAS datasets, our analyses for some of the etiologically related traits remain underpowered. Finally, our analyses were primarily based on datasets involving individuals of European ancestry and might thus not be applicable to other ethnicities.

In conclusion, our results suggest causal associations of T2D and hyperglycemia with a higher risk for ischemic stroke, particularly large artery and small vessel stroke. Against findings from secondary analyses of clinical trials, our results support that therapeutic approaches aimed at lowering $\mathrm{HbA} 1 \mathrm{c}$ have the potential to decrease the risk of ischemic stroke. The effects of genetically determined insulin resistance and $\beta$-cell dysfunction on large artery and small vessel stroke observed here might have implications for anti-diabetic treatments specifically targeting these mechanisms.

Acknowledgements: This research has been conducted using the UK Biobank Resource (UK Biobank application 2532). We acknowledge the contributions by the DIAGRAM Consortium, the MAGIC Consortium, the MEGASTROKE Consortium, the ISGC Consortium, and the CHARGE Consortium for making their data publicly available. MEGASTROKE has received funding from the sources detailed at http://www.megastroke.org/acknowledgments.html.

Sources of funding: M. Georgakis is funded by a scholarship from the Onassis Foundation. This project has received funding from the European Union's Horizon 2020 research and innovation programme (No 666881), SVDs@ @target (to M. Dichgans) and No 667375, CoSTREAM (to M. Dichgans and H. S. Markus); the DFG as part of the Munich Cluster for Systems Neurology (EXC 1010 SyNergy) and the CRC 1123 (B3) (to M. Dichgans); the Corona Foundation (to M. Dichgans); the Fondation Leducq (Transatlantic Network of Excellence on the Pathogenesis of Small Vessel Disease of the Brain) (to M. Dichgans); a grant for strategic collaboration between LMU Munich and Cambridge University; British Heart Foundation Programme Grant RG/16/4/32218 (To H. Markus); 
Infratrsuctural support from the Cambridge Univeristy Hospitals NIHR Comprehensive Biomedical Research Centre.

Author contributions: MKG, EH, RM, HSM, and MD conceived and designed the study. MKG, $\mathrm{EH}$, and RM analysed the data. All authors participated in interpretation of results. MKG and MD drafted the manuscript. All authors critically revised the manuscript for intellectual content. All authors approved the submitted version and are accountable for the integrity of this work.

Disclosures: None.

\section{REFERENCES}

1. Collaborators GBDLRoS, Feigin VL, Nguyen G, et al. Global, regional, and country-specific lifetime risks of stroke, 1990 and 2016. N Engl J Med. 2018;379:2429-2437

2. DALYs GBD, Collaborators H. Global, regional, and national disability-adjusted life-years (dalys) for 315 diseases and injuries and healthy life expectancy (hale), 1990-2015: A systematic analysis for the global burden of disease study 2015. Lancet. 2016;388:1603-1658

3. Mortality GBD, Causes of Death C. Global, regional, and national life expectancy, all-cause mortality, and cause-specific mortality for 249 causes of death, 1980-2015: A systematic analysis for the global burden of disease study 2015. Lancet. 2016;388:1459-1544

4. Cosentino F, Grant PJ, Aboyans V, et al. 2019 esc guidelines on diabetes, pre-diabetes, and cardiovascular diseases developed in collaboration with the easd. Eur Heart J. 2019

5. Meschia JF, Bushnell C, Boden-Albala B, et al. Guidelines for the primary prevention of stroke: A statement for healthcare professionals from the american heart association/american stroke association. Stroke. 2014;45:3754-3832

6. Emerging Risk Factors C, Sarwar N, Gao P, et al. Diabetes mellitus, fasting blood glucose concentration, and risk of vascular disease: A collaborative meta-analysis of 102 prospective studies. Lancet. 2010;375:2215-2222

7. Mitsios JP, Ekinci EI, Mitsios GP, Churilov L, Thijs V. Relationship between glycated hemoglobin and stroke risk: A systematic review and meta-analysis. J Am Heart Assoc. 2018;7

8. Fang HJ, Zhou YH, Tian YJ, Du HY, Sun YX, Zhong LY. Effects of intensive glucose lowering in treatment of type 2 diabetes mellitus on cardiovascular outcomes: A meta-analysis of data from 58,160 patients in 13 randomized controlled trials. Int J Cardiol. 2016;218:50-58

9. Group AC, Patel A, MacMahon S, et al. Intensive blood glucose control and vascular outcomes in patients with type 2 diabetes. $N$ Engl J Med. 2008;358:2560-2572

10. Action to Control Cardiovascular Risk in Diabetes Study G, Gerstein HC, Miller ME, Byington RP, Goff DC, Jr., Bigger JT, Buse JB, Cushman WC, Genuth S, Ismail-Beigi F, Grimm RH, Jr., Probstfield JL, Simons-Morton DG, Friedewald WT. Effects of intensive glucose lowering in type 2 diabetes. N Engl J Med. 2008;358:2545-2559

11. Ray KK, Seshasai SR, Wijesuriya S, Sivakumaran R, Nethercott S, Preiss D, Erqou S, Sattar N. Effect of intensive control of glucose on cardiovascular outcomes and death in patients with diabetes mellitus: A meta-analysis of randomised controlled trials. Lancet. 2009;373:1765-1772

12. Tahrani AA, Barnett AH, Bailey CJ. Pharmacology and therapeutic implications of current drugs for type 2 diabetes mellitus. Nat Rev Endocrinol. 2016;12:566-592

13. Imamura F, Mukamal KJ, Meigs JB, Luchsinger JA, Ix JH, Siscovick DS, Mozaffarian D. Risk factors for type 2 diabetes mellitus preceded by beta-cell dysfunction, insulin resistance, or both in older adults: The cardiovascular health study. Am J Epidemiol. 2013;177:1418-1429

14. Zethelius B, Byberg L, Hales CN, Lithell H, Berne C. Proinsulin is an independent predictor of coronary heart disease: Report from a 27-year follow-up study. Circulation. 2002;105:2153-2158 
15. Gast KB, Tjeerdema N, Stijnen T, Smit JW, Dekkers OM. Insulin resistance and risk of incident cardiovascular events in adults without diabetes: Meta-analysis. PLoS One. 2012;7:e52036

16. Holmes MV, Ala-Korpela M, Smith GD. Mendelian randomization in cardiometabolic disease: Challenges in evaluating causality. Nature reviews. Cardiology. 2017;14:577-590

17. O'Donnell CJ, Sabatine MS. Opportunities and challenges in mendelian randomization studies to guide trial design. JAMA Cardiol. 2018;3:967

18. Georgakis MK, Gill D, Rannikmae K, Traylor M, Anderson CD, Lee JM, Kamatani Y, Hopewell JC, Worrall BB, Bernhagen J, Sudlow CLM, Malik R, Dichgans M. Genetically determined levels of circulating cytokines and risk of stroke. Circulation. 2019;139:256-268

19. Davey Smith G, Davies NM, Dimou N, et al. Strobe-mr: Guidelines for strengthening the reporting of mendelian randomization studies. . PeerJ Preprints. 2019;7:e27857v27851

20. Mahajan A, Taliun D, Thurner M, et al. Fine-mapping type 2 diabetes loci to single-variant resolution using high-density imputation and islet-specific epigenome maps. Nat Genet. 2018;50:1505-1513

21. Sudlow C, Gallacher J, Allen N, et al. Uk biobank: An open access resource for identifying the causes of a wide range of complex diseases of middle and old age. PLoS Med. 2015;12:e1001779

22. Scott RA, Lagou V, Welch RP, et al. Large-scale association analyses identify new loci influencing glycemic traits and provide insight into the underlying biological pathways. Nat Genet. 2012;44:9911005

23. Wheeler E, Leong A, Liu CT, et al. Impact of common genetic determinants of hemoglobin a1c on type 2 diabetes risk and diagnosis in ancestrally diverse populations: A transethnic genome-wide meta-analysis. PLoS Med. 2017;14:e1002383

24. Kamat MA, Blackshaw JA, Young R, Surendran P, Burgess S, Danesh J, Butterworth AS, Staley JR. Phenoscanner v2: An expanded tool for searching human genotype-phenotype associations. Bioinformatics. 2019

25. Heid IM, Winkler TW. A multitrait gwas sheds light on insulin resistance. Nat Genet. 2016;49:7-8

26. Strawbridge RJ, Dupuis J, Prokopenko I, et al. Genome-wide association identifies nine common variants associated with fasting proinsulin levels and provides new insights into the pathophysiology of type 2 diabetes. Diabetes. 2011;60:2624-2634

27. Li M, Feng D, Zhang K, Gao S, Lu J. Disproportionately elevated proinsulin levels as an early indicator of beta-cell dysfunction in nondiabetic offspring of chinese diabetic patients. Int J Endocrinol. 2016;2016:4740678

28. Pfutzner A, Forst T. Elevated intact proinsulin levels are indicative of beta-cell dysfunction, insulin resistance, and cardiovascular risk: Impact of the antidiabetic agent pioglitazone. J Diabetes Sci Technol. 2011;5:784-793

29. Udler MS, McCarthy MI, Florez JC, Mahajan A. Genetic risk scores for diabetes diagnosis and precision medicine. Endocr Rev. 2019;40:1500-1520

30. Udler MS, Kim J, von Grotthuss M, et al. Type 2 diabetes genetic loci informed by multi-trait associations point to disease mechanisms and subtypes: A soft clustering analysis. PLoS Med. 2018; $15: \mathrm{e} 1002654$

31. So HC, Gui AH, Cherny SS, Sham PC. Evaluating the heritability explained by known susceptibility variants: A survey of ten complex diseases. Genet Epidemiol. 2011;35:310-317

32. Shim H, Chasman DI, Smith JD, Mora S, Ridker PM, Nickerson DA, Krauss RM, Stephens M. A multivariate genome-wide association analysis of $10 \mathrm{ldl}$ subfractions, and their response to statin treatment, in 1868 caucasians. PLoS One. 2015;10:e0120758

33. International Diabetes Federation. Idf diabetes atlas - 7 th edition. Diabetesatlas http://www.Diabetesatlas.Org/ (2015).

34. Palmer TM, Lawlor DA, Harbord RM, Sheehan NA, Tobias JH, Timpson NJ, Davey Smith G, Sterne JA. Using multiple genetic variants as instrumental variables for modifiable risk factors. Stat Methods Med Res. 2012;21:223-242

35. Malik R, Chauhan G, Traylor M, et al. Multiancestry genome-wide association study of 520,000 subjects identifies 32 loci associated with stroke and stroke subtypes. Nat Genet. 2018;50:524-537

36. Malik R, Rannikmae K, Traylor M, Georgakis MK, Sargurupremraj M, Markus HS, Hopewell JC, Debette S, Sudlow CLM, Dichgans M, consortium M, the International Stroke Genetics C. Genomewide meta-analysis identifies 3 novel loci associated with stroke. Ann Neurol. 2018;84:934-939

37. Adams HP, Jr., Bendixen BH, Kappelle LJ, Biller J, Love BB, Gordon DL, Marsh EE, 3rd. Classification of subtype of acute ischemic stroke. Definitions for use in a multicenter clinical trial. Toast. Trial of org 10172 in acute stroke treatment. Stroke. 1993;24:35-41

38. Woo D, Falcone GJ, Devan WJ, et al. Meta-analysis of genome-wide association studies identifies 1q22 as a susceptibility locus for intracerebral hemorrhage. Am J Hum Genet. 2014;94:511-521 
39. Franceschini N, Giambartolomei C, de Vries PS, et al. Gwas and colocalization analyses implicate carotid intima-media thickness and carotid plaque loci in cardiovascular outcomes. Nat Commun. 2018;9:5141

40. Cox SR, Lyall DM, Ritchie SJ, et al. Associations between vascular risk factors and brain mri indices in uk biobank. Eur Heart J. 2019;40:2290-2300

41. Burgess S, Butterworth A, Thompson SG. Mendelian randomization analysis with multiple genetic variants using summarized data. Genet Epidemiol. 2013;37:658-665

42. Bowden J, Hemani G, Davey Smith G. Invited commentary: Detecting individual and global horizontal pleiotropy in mendelian randomization-a job for the humble heterogeneity statistic? Am J Epidemiol. 2018;187:2681-2685

43. Hartwig FP, Davey Smith G, Bowden J. Robust inference in summary data mendelian randomization via the zero modal pleiotropy assumption. International journal of epidemiology. 2017;46:1985-1998

44. Bowden J, Davey Smith G, Burgess S. Mendelian randomization with invalid instruments: Effect estimation and bias detection through egger regression. International journal of epidemiology. 2015;44:512-525

45. Verbanck M, Chen CY, Neale B, Do R. Detection of widespread horizontal pleiotropy in causal relationships inferred from mendelian randomization between complex traits and diseases. Nat Genet. 2018;50:693-698

46. Burgess S, Daniel RM, Butterworth AS, Thompson SG, Consortium E-IA. Network mendelian randomization: Using genetic variants as instrumental variables to investigate mediation in causal pathways. Int J Epidemiol. 2015;44:484-495

47. Burgess S, Thompson SG. Multivariable mendelian randomization: The use of pleiotropic genetic variants to estimate causal effects. Am J Epidemiol. 2015;181:251-260

48. Carter AR, Sanderson E, Hammerton G, Richmond RC, Smith GD, Heron J, Taylor AE, Davies NM, Howe LD. Mendelian randomisation for mediation analysis: Current methods and challenges for implementation. bioRxiv. 2019:835819

49. Larsson SC, Scott RA, Traylor M, Langenberg CC, Hindy G, Melander O, Orho-Melander M, Seshadri S, Wareham NJ, Markus HS, Collaboration M, Network NSG. Type 2 diabetes, glucose, insulin, bmi, and ischemic stroke subtypes: Mendelian randomization study. Neurology. 2017;89:454460

50. Liu J, Rutten-Jacobs L, Liu M, Markus HS, Traylor M. Causal impact of type 2 diabetes mellitus on cerebral small vessel disease: A mendelian randomization analysis. Stroke. 2018;49:1325-1331

51. Bonnet F, Scheen AJ. Impact of glucose-lowering therapies on risk of stroke in type 2 diabetes. Diabetes Metab. 2017;43:299-313

52. Lim S, Oh TJ, Dawson J, Sattar N. Diabetes drugs and stroke risk: Intensive versus conventional glucose-lowering strategies, and implications of recent cardiovascular outcome trials. Diabetes Obes Metab. 2019

53. Wardlaw JM, Smith EE, Biessels GJ, et al. Neuroimaging standards for research into small vessel disease and its contribution to ageing and neurodegeneration. Lancet Neurol. 2013;12:822-838

54. Dupuis J, Langenberg C, Prokopenko I, et al. New genetic loci implicated in fasting glucose homeostasis and their impact on type 2 diabetes risk. Nat Genet. 2010;42:105-116

55. Holmes MV, Davey Smith G. Problems in interpreting and using gwas of conditional phenotypes illustrated by 'alcohol gwas'. Mol Psychiatry. 2019;24:167-168 
Table 1. Data sources that were used in the analyses for the current study.

\begin{tabular}{|c|c|c|c|c|c|}
\hline Phenotype & Source & $\begin{array}{l}\mathbf{N} \text { (Total or } \\
\text { Cases/Controls }\end{array}$ & $\begin{array}{l}\text { Imputation } \\
\text { reference panel }\end{array}$ & Ancestry & Adjustments \\
\hline Diabetes mellitus type 2 & DIAGRAM Consortium $^{20}$ & $74,124 / 824,006$ & $\mathrm{HRC}$ & European & age, sex, population structure \\
\hline $\mathrm{HbA1c}$ & UK Biobank $^{21}$ & 408,989 & $\mathrm{HRC}+\mathrm{UK} 10 \mathrm{~K}$ & White British & $\begin{array}{l}\text { age, sex, population structure, } \\
\text { genotyping platform array, assessment } \\
\text { center }\end{array}$ \\
\hline Fasting glucose levels & MAGIC Consortium $^{22}$ & 133,010 & HapMap & European & age, sex, population structure \\
\hline $\begin{array}{l}\text { Insulin resistance (fasting } \\
\text { insulin levels) }\end{array}$ & $\begin{array}{l}\text { Multi-trait GWAS }{ }^{25} \text { and MAGIC } \\
\text { Consortium }^{22}\end{array}$ & 108,557 & HapMap & European & age, sex, BMI, population structure \\
\hline $\begin{array}{l}\beta \text {-cell dysfunction (fasting } \\
\text { proinsulin levels) }\end{array}$ & MAGIC Consortium $^{26}$ & 16,378 & 1000 Genomes & European & $\begin{array}{l}\text { age, sex, fasting insulin, population } \\
\text { structure }\end{array}$ \\
\hline Any ischemic stroke & MEGASTROKE Consortium ${ }^{35}$ & $60,341 / 454,450$ & 1000 Genomes & Trans-ethnic (70\% European) & age, sex, population structure \\
\hline Large artery stroke & MEGASTROKE Consortium ${ }^{35}$ & $6,688 / 454,450$ & 1000 Genomes & Trans-ethnic (70\% European) & age, sex, population structure \\
\hline Cardioembolic stroke & MEGASTROKE Consortium ${ }^{35}$ & $9,006 / 454,450$ & 1000 Genomes & Trans-ethnic (70\% European) & age, sex, population structure \\
\hline Small vessel stroke & MEGASTROKE Consortium ${ }^{35}$ & $11,710 / 454,450$ & 1000 Genomes & Trans-ethnic (70\% European) & age, sex, population structure \\
\hline Intracerebral hemorrhage & ISGC meta-analysis $^{38}$ & $1,545 / 1,481$ & 1000 Genomes & European & age, sex, population structure \\
\hline Carotid plaque & CHARGE Consortium $^{39}$ & $21,540 / 26,894$ & 1000 Genomes & European & age, sex, population structure \\
\hline WMH volume & UK Biobank imaging database ${ }^{40}$ & 17,534 & $\mathrm{HRC}+\mathrm{UK} 10 \mathrm{~K}$ & White British & $\begin{array}{l}\text { age, sex, mean resting and task } \\
\text { functional MRI head motion, population } \\
\text { structure, genotyping platform array }\end{array}$ \\
\hline Mean diffusivity & UK Biobank imaging database $\mathrm{e}^{40}$ & 17,534 & $\mathrm{HRC}+\mathrm{UK} 10 \mathrm{~K}$ & White British & $\begin{array}{l}\text { age, sex, mean resting and task } \\
\text { functional MRI head motion, population } \\
\text { structure, genotyping platform array }\end{array}$ \\
\hline Fractional anisotropy & UK Biobank imaging database ${ }^{40}$ & 17,534 & $\mathrm{HRC}+\mathrm{UK} 10 \mathrm{~K}$ & White British & $\begin{array}{l}\text { age, sex, mean resting and task } \\
\text { functional MRI head motion, population } \\
\text { structure, genotyping platform array }\end{array}$ \\
\hline $\begin{array}{l}\text { Normalized grey matter } \\
\text { volume }\end{array}$ & UK Biobank imaging database ${ }^{40}$ & 17,534 & $\mathrm{HRC}+\mathrm{UK} 10 \mathrm{~K}$ & White British & $\begin{array}{l}\text { age, sex, mean resting and task } \\
\text { functional MRI head motion, population } \\
\text { structure, genotyping platform array }\end{array}$ \\
\hline $\begin{array}{l}\text { Normalized total brain } \\
\text { volume }\end{array}$ & UK Biobank imaging database ${ }^{40}$ & 17,534 & $\mathrm{HRC}+\mathrm{UK} 10 \mathrm{~K}$ & White British & $\begin{array}{l}\text { age, sex, mean resting and task } \\
\text { functional MRI head motion, population } \\
\text { structure, genotyping platform array }\end{array}$ \\
\hline
\end{tabular}


Table 2. Mendelian randomization associations between genetically determined diabetic traits and etiologically related cerebrovascular phenotypes, as derived from random-effects inverse-variance weighted analyses.

\begin{tabular}{|c|c|c|c|c|c|c|}
\hline \multirow[b]{2}{*}{ Exposure } & \multirow[b]{2}{*}{$\begin{array}{c}\text { Carotid plaque } \\
\text { OR }(95 \% \mathrm{CI}) \\
\end{array}$} & \multicolumn{3}{|c|}{ White matter integrity } & \multicolumn{2}{|c|}{ Brain atrophy } \\
\hline & & WMH volume & Mean diffusivity & $\begin{array}{c}\text { Fractional anisotropy } \\
\text { beta }(95 \% \mathrm{CI}) \\
\end{array}$ & Grey matter volume & Total brain volume \\
\hline Diabetes mellitus type 2 & $1.08(1.04-1.12)$ & $0.003(-0.016,0.023)$ & $0.011(-0.011,0.033)$ & $-0.028(-0.048,-0.006)$ & $-0.023(-0.041,-0.005)^{\mathrm{a}}$ & $-0.021(-0.041,-0.001)^{\mathrm{a}}$ \\
\hline HbA1c (1\%-increment) & $1.52(1.24-1.85)$ & $0.047(-0.071,0.164)$ & $0.002(-0.129,0.133)$ & $-0.091(-0.206,0.025)$ & $-0.122(-0.230,-0.014)^{a}$ & $-0.083(-0.204,0.039$ \\
\hline $\begin{array}{l}\text { Fasting glucose levels ( } 1 \mathrm{SD} \\
\text { increment) }\end{array}$ & $1.16(0.93-1.44)$ & $-0.012(-0.135,0.111)$ & $-0.060(-0.175,0.055)$ & $-0.034(-0.158,0.090)$ & $-0.072(-0.191,0.048)$ & $-0.092(-0.212,0.027)$ 总 \\
\hline $\begin{array}{l}\text { Insulin resistance ( } 1 \text { log-increment } \\
\text { in fasting insulin levels) }\end{array}$ & $0.93(0.83-1.05)$ & $0.094(-0.062,0.251)$ & $0.146(-0.056,0.347)$ & $-0.181(-0.380,0.019)$ & $-0.039(-0.220,0.142)$ & $-0.087(-0.285,0.112) \frac{\overline{\frac{D}{D}}}{\frac{\overline{0}}{\Phi}}$ \\
\hline $\begin{array}{l}\text { B-cell dysfunction (1 log-increment } \\
\text { in fasting proinsulin levels) }\end{array}$ & $1.10(0.80-1.50)$ & $0.062(-0.021,0.146)$ & $0.048(-0.017,0.114)$ & $-0.048(-0.115,0.020)$ & $-0.130(-0.195,-0.065)$ & $-0.170(-0.232,-0.108)$ \\
\hline
\end{tabular}

Odds Ratios (OR) are presented for binary traits (carotid plaque) and beta coefficients (standardized based on the SD of the measure) for the continuous imaging traits.

Bold indicates statistical significance at an FDR-adjusted p-value $<0.05$.

${ }^{a}$ Associations reaching nominal significance (unadjusted $\mathrm{p}<0.05$ ) 
Figure 1. Schematic representation of our study design and assumptions of Mendelian randomization (MR) analyses. The assumptions of the MR study design include the following: the genetic variants (instruments) must be associated with the exposure (assumption 1); the variants must not be associated with confounders (assumption 2); the variants must influence the outcome only through the risk factor under study (assumption 3).

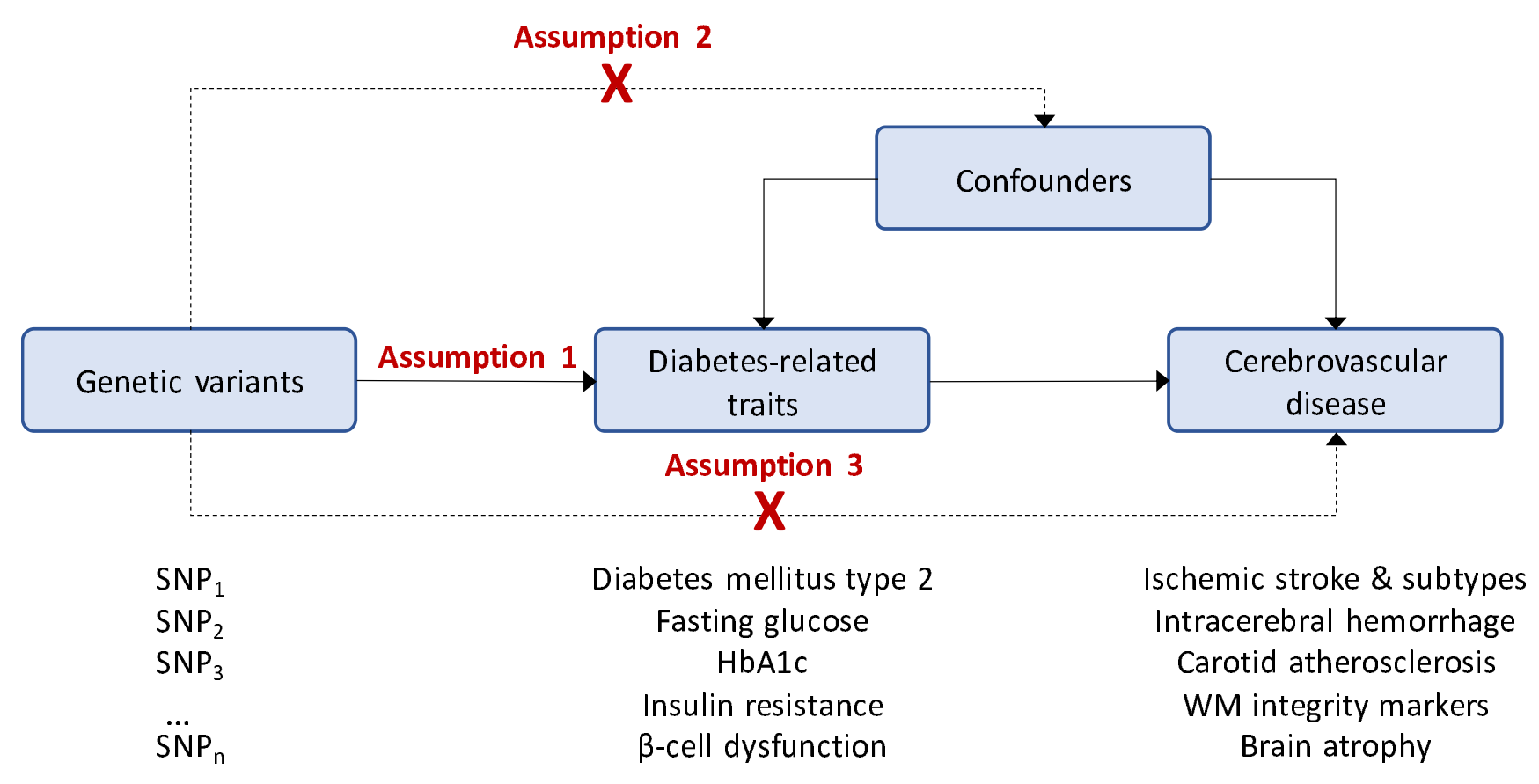

Abbreviations. HbA1c, Glycated hemoglobin; SNP, single nucleotide polymorphism; WM, white matter. 
medRxiv preprint doi: https://doi.org/10.1101/2019.12.27.19015834; this version posted December 30, 2019. The copyright holder for this preprint (which was not certified by peer review) is the author/funder, who has granted medRxiv a license to display the preprint in perpetuity.

It is made available under a CC-BY 4.0 International license .

Figure 2. Mendelian Randomization associations between genetic predisposition to type 2 diabetes mellitus and stroke subtypes. Results derived from random-effects inverse-variance weighted analyses.

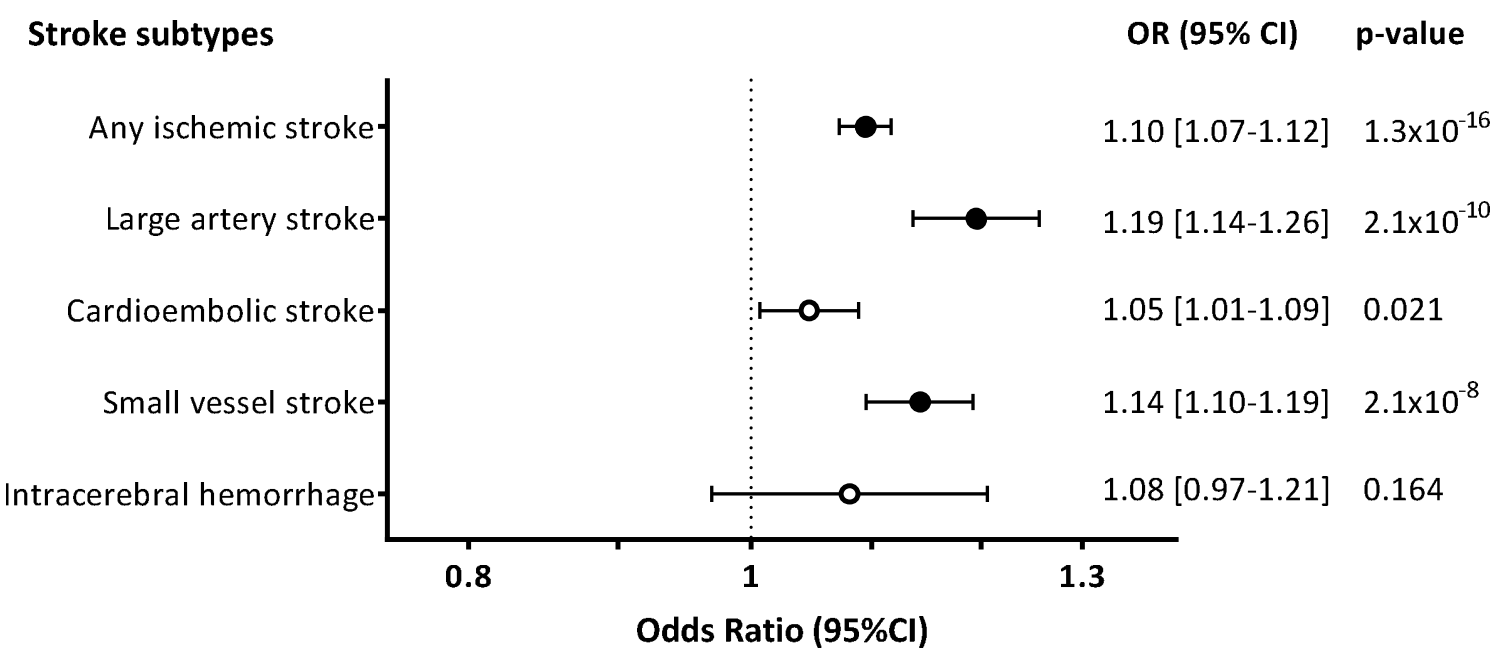

Full circles correspond to statistically significant association estimates at an FDR-adjusted p-value $<0.05$. 
Figure 3. Mendelian Randomization associations of genetically determined hyperglycemia traits (HbA1c and fasting glucose) with stroke subtypes. Results derived from random-effects inversevariance weighted analyses.

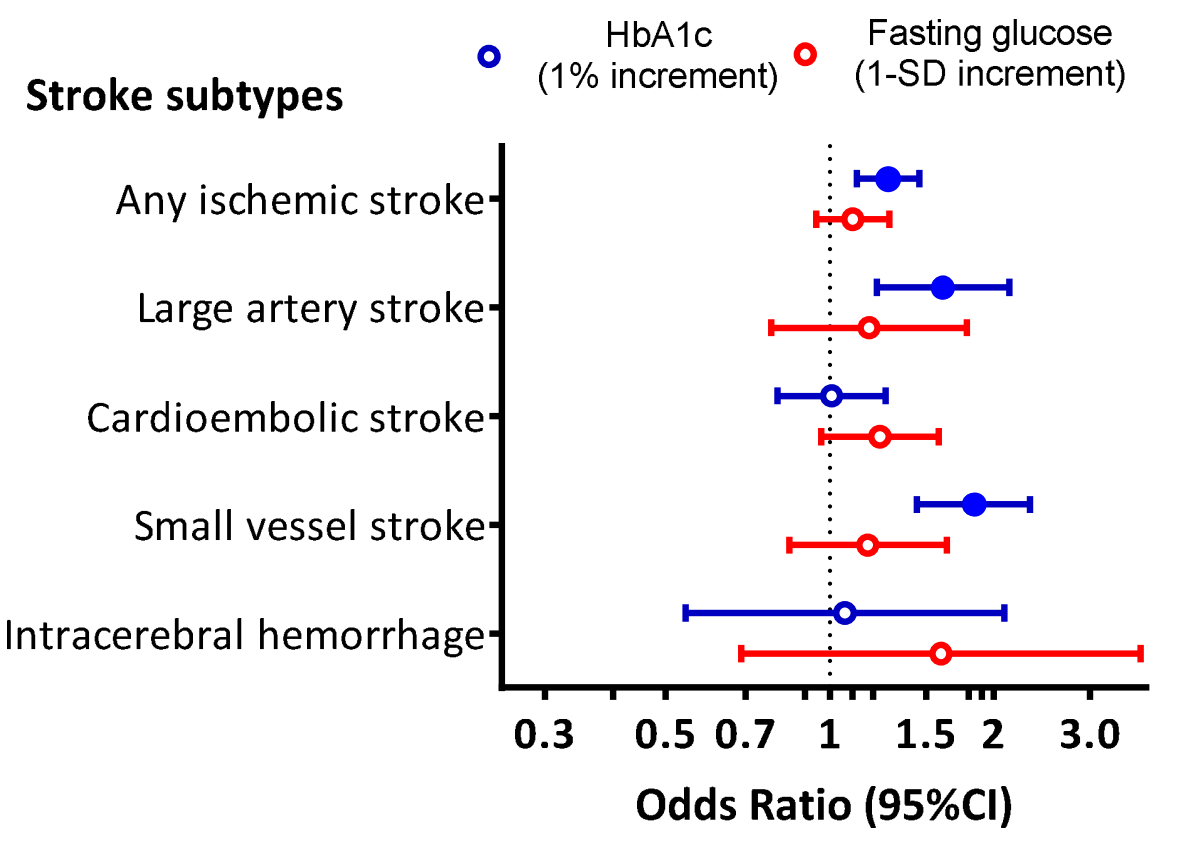

Full colored circles correspond to statistically significant association estimates at an FDR-adjusted p-value $<0.05$. Abbreviations. HbA1c, Glycated hemoglobin. 
medRxiv preprint doi: https://doi.org/10.1101/2019.12.27.19015834; this version posted December 30, 2019. The copyright holder for this preprint (which was not certified by peer review) is the author/funder, who has granted medRxiv a license to display the preprint in perpetuity.

It is made available under a CC-BY 4.0 International license .

Figure 4. Mendelian Randomization associations of genetically determined insulin resistance and B-cell dysfunction with stroke subtypes. (A) Results derived from random-effects inverse-variance weighted analyses. (B) Heatmap of the associations between clusters of diabetic endophenotypes related to $\beta$-cell dysfunction and insulin resistance with the risk of stroke subtypes.

A - Insulin resistance $\beta$-cell dysfunction
(fasting proinsulin, 1-log increment)

\section{Stroke subtypes}

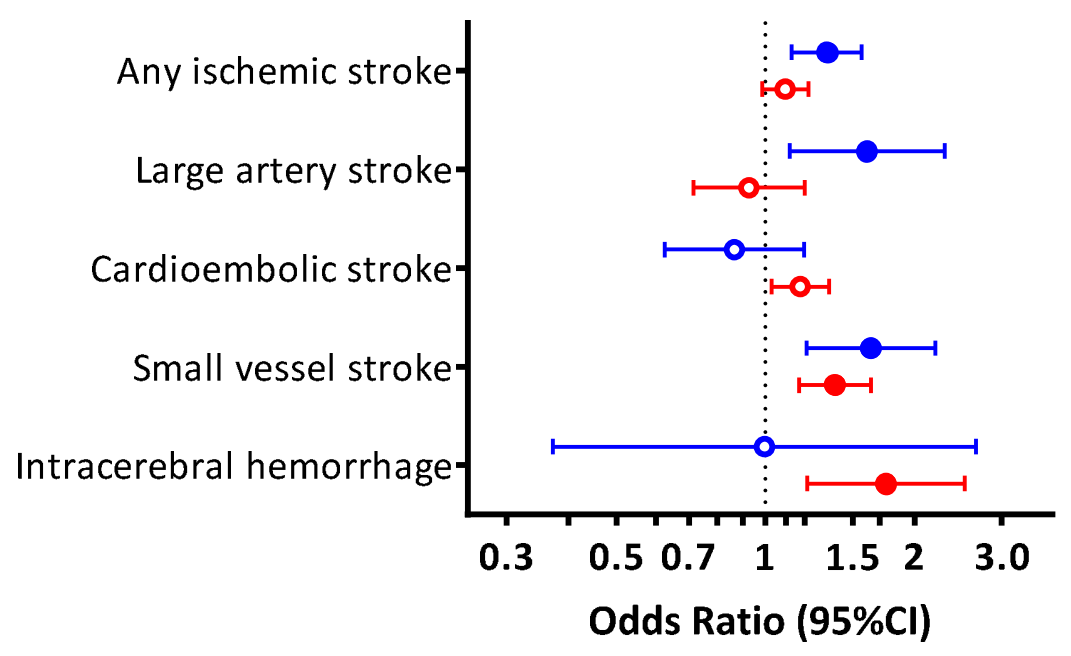

B

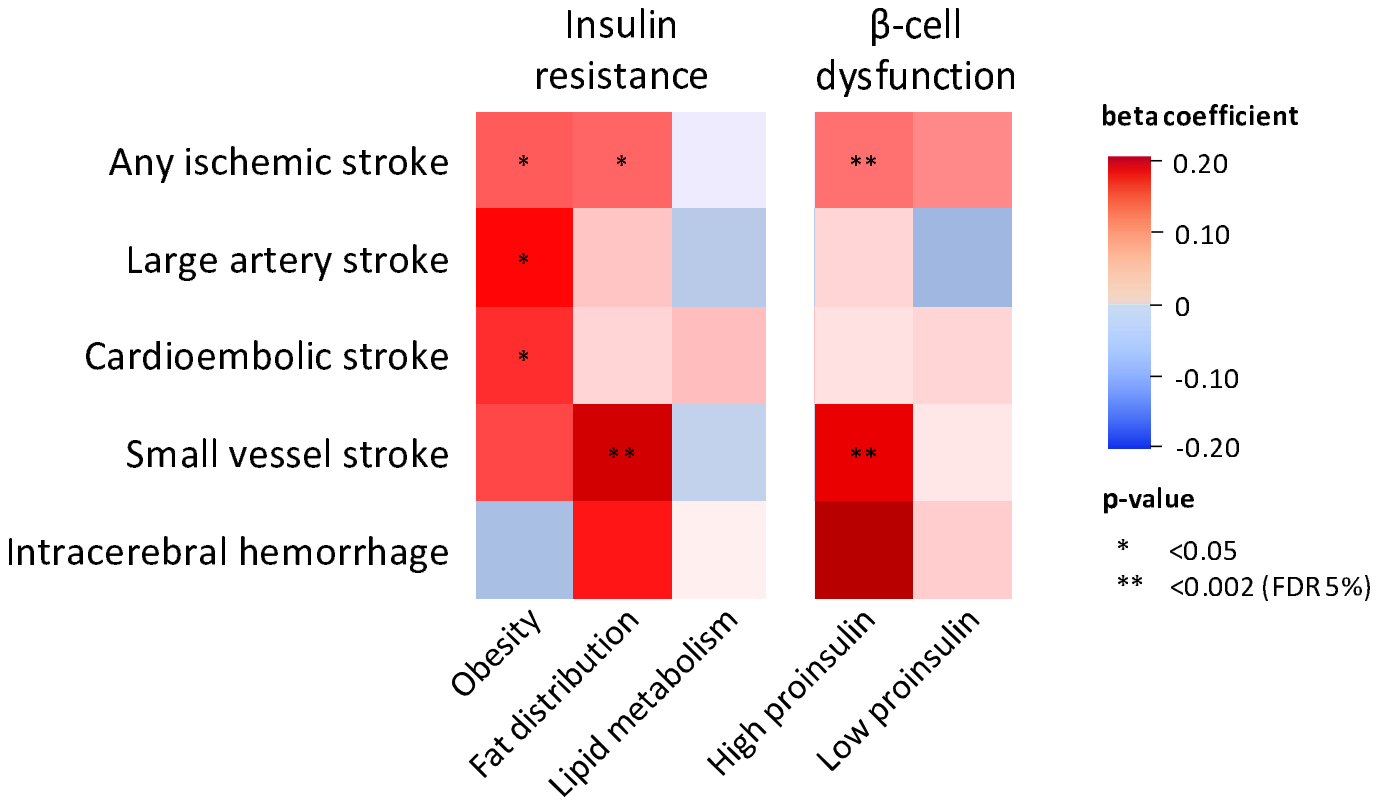

Full colored circles in panel A correspond to statistically significant association estimates at an FDR-adjusted pvalue $<0.05$. 
Figure 5. Mendelian randomization (MR)-based mediation analyses between genetically predicted insulin resistance, $\beta$-cell dysfunction, HbA1c levels and risk of large artery and small vessel stroke. (A, B) Direct and indirect (through HbAlc levels) effects of insulin resistance and $\beta$-cell dysfunction and effects of HbA1c adjusted for these phenotypes (by multivariable MR) on risk of large artery and small vessel stroke. (C, B) Schematic representation of the results.

A

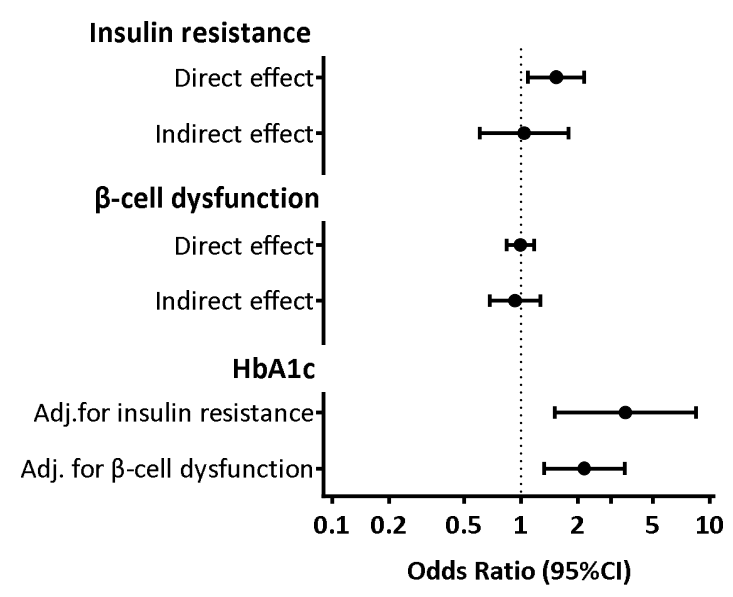

C

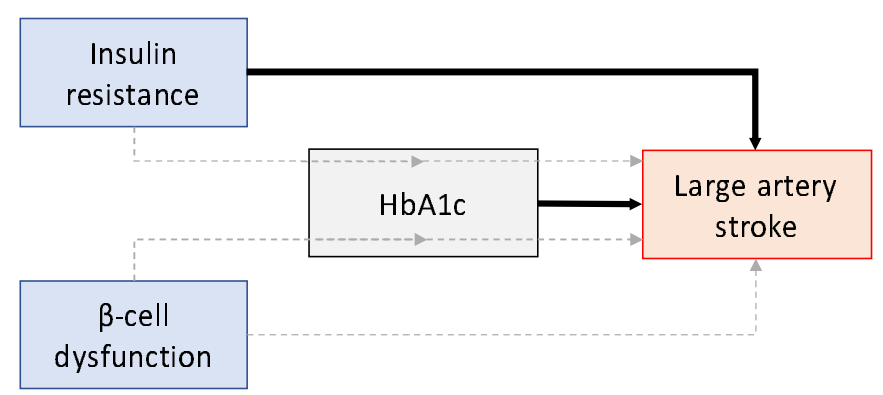

B

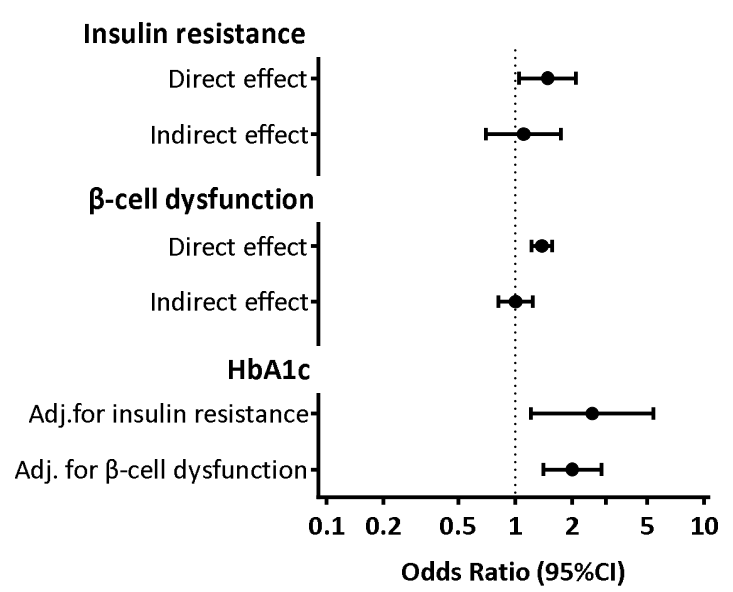

D

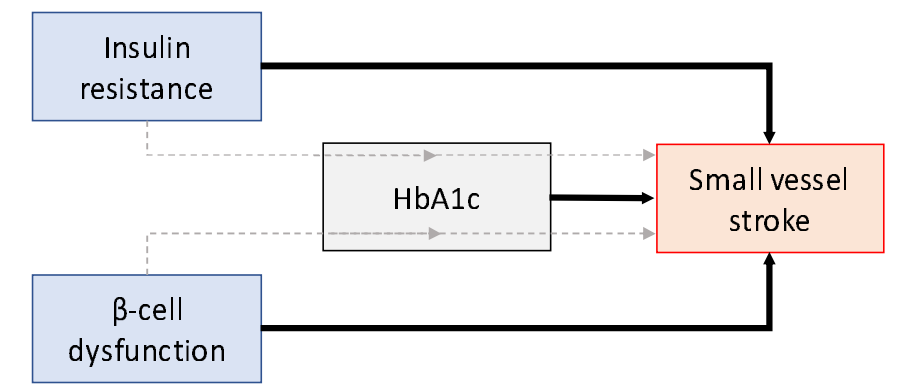

Dark arrows represent significant associations, whereas light grey dotted lines represent non-significant associations. Abbreviations. HbA1c, Glycated hemoglobin A1c. 